\title{
Observation and Modelling of the Onset of Parametric Decay Instabilities dur- ing Gyrotron Operation at ASDEX Upgrade
}

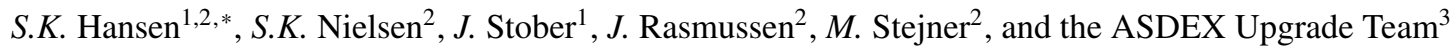 \\ ${ }^{1}$ Max-Planck-Institut für Plasmaphysik, Boltzmannstraße, D-85748 Garching b. München, Germany \\ ${ }^{2}$ Department of Physics, Technical University of Denmark, Fysikvej, DK-2800 Kgs. Lyngby, Denmark \\ ${ }^{3}$ See Appendix in A. Kallenbach for the ASDEX Upgrade Team and the EUROfusion MST1 Team, Nucl. Fusion 57, 102015 (2017)
}

\begin{abstract}
We investigate parametric decay instabilities (PDIs) occurring for gyrotron radiation near the upper hybrid resonance at the ASDEX Upgrade tokamak. The PDIs are observed through anomalous millimeter-wave scattering which is recorded using the high-resolution, fast acquisition collective Thomson scattering system installed at ASDEX Upgrade, and an experiment in which such observations are made during a scan of the toroidal magnetic field is performed. A previously published theoretical model is used to calculate the gyrotron power necessary to excite PDIs in the experiment; the theoretical model is capable of predicting whether or not PDIs will be observed at a given toroidal magnetic field with a high degree of accuracy.
\end{abstract}

\section{Introduction}

When high-power gyrotron beams are injected into magnetically confined fusion plasmas, the wave amplitude may in some cases become so large that the linear approximation breaks down and a parametric decay instability (PDI), which couples the incoming electromagnetic wave to two (electrostatic) plasma waves, is excited. Energy and momentum conservation in the three-wave process require the selection rules,

$$
\omega_{0}=\omega_{1}+\omega_{2}, \quad \mathbf{k}_{0}=\mathbf{k}_{1}+\mathbf{k}_{2},
$$

to be satisfied; here, $\omega$ is the angular frequency, $\mathbf{k}$ is the wave vector, subscript 0 refers to the gyrotron wave, and subscripts 1,2 refer to the plasma waves excited by the PDI. In the cases of interest to us, the wave referred to by subscript 1 is a low-frequency wave whose frequency lies near the lower hybrid (LH) frequency, $\omega_{L H}$, while the wave referred to by subscript 2 is a high-frequency wave whose frequency is close to that of the gyrotron. Because of the frequency selection rule, the high-frequency wave will be down-shifted by approximately $\omega_{L H}(\sim 2 \pi \times 1 \mathrm{GHz}$ in the main plasma of ASDEX Upgrade) from the gyrotron radiation, generally placing it outside the notch filters protecting the detectors used for millimeter-wave diagnostics such as ECE and collective Thomson scattering (CTS), causing it to hamper or potentially even damage these diagnostics. It is thus critical to understand PDIs in order to mitigate their potentially deleterious effects and, ideally, exploit the diagnostic tools that may be provided by them.

In this paper, we investigate PDIs occurring for Xmode radiation near the upper hybrid resonance (UHR), where $\omega_{0}=\omega_{U H}$, with $\omega_{U H}$ being the UH frequency. PDIs

*e-mail: Soeren.Kjer.Hansen@ipp.mpg.de are likely to occur for X-mode radiation near the UHR due to field enhancement effects, as noted by [1], who particularly considered the case of decay of the X-mode wave to a warm LH wave and an electron Bernstein wave, in connection with X1 ECRH of an optically thin resonance. The occurrence of PDIs in connection with X1 ECRH was observed in a number of small toroidal devices with optically thin resonances, e.g. Versator II [2], FT-1 [3], and TCA [4]. Such PDIs were also observed in connection with O1 ECRH at W VII-A [5] and TCA [4], which was attributed to conversion of a significant amount of power to X-mode upon reflection from the vessel walls; these observations indicate that the PDIs may also be of relevance during the start-up phase in ITER. The latter mechanism has also been used to explain PDIs observed during CTS experiments at ASDEX Upgrade [6-8], where the ECR is optically thin due to its location far on the high-field side in order to minimize the ECE background, and may also explain similar observations from LHD [9]. Besides the above cases, PDIs near the UHR have been shown to occur in connection with $\mathrm{O}-\mathrm{X}-\mathrm{B}$ heating of overdense plasmas, e.g. at W-7AS [10, 11], MAST [12], and TJ-K [13]; such PDIs may also be expected in connection with $\mathrm{O}-\mathrm{X}$ $\mathrm{B}$ heating at W-7X.

In the present work, we seek to validate the theoretical model from [8], which gives the gyrotron power threshold, $P_{0}^{\text {th }}$, that must be exceeded in order to excite PDIs in the CTS experiments at ASDEX Upgrade, by calculating the fraction of power converted to X-mode upon the reflection from the wall, the optical thickness of the ECR, the field enhancement near the UHR, and assuming decay of the X-mode wave to a warm LH wave and an electron Bernstein wave. To do this, O-mode pulses with a maximum power, $\max \left(P_{0}\right)$, of approximately $300 \mathrm{~kW}$ 
and $\omega_{0}=2 \pi \times 105 \mathrm{GHz}$ are injected by a gyrotron from to the system described in [14], and the electromagnetic emission/scattering in $\mathrm{O}$-mode at frequencies close to $\omega_{0}$ is measured during the pulses using the fast acquisition CTS system presented in [15]. From the above observations, the occurrence of PDI features in a given pulse can be established and compared with whether or not $\max \left(P_{0}\right)$ exceeds $P_{0}^{\text {th }}$ from [8], thus allowing the predictive value of the theoretical model to be assessed.

The paper is arranged as follows: in Section 2 the experimental setup and observations at ASDEX Upgrade are described; in Section 3 the results of the theoretical modelling are presented and compared with the observations; finally, in Section 4 we draw our conclusions.

\section{Observations}

The observations presented in this paper stem from ASDEX Upgrade discharge \#34575, which is an H-mode deuterium discharge, during which the toroidal magnetic field, $B_{t}$, is scanned from $-2.66 \mathrm{~T}$ to $-2.43 \mathrm{~T}$; for reference, the central electron density and temperature in the discharge are approximately $5 \times 10^{19} \mathrm{~m}^{-3}$ and $4 \mathrm{keV}$, respectively. The most important effect of the $B_{t}$-scan for our purposes is the displacement of the ECR from inside the main plasma to the high-field side scrape-off layer which increases the fraction of power transmitted through the ECR by an order of magnitude, as will be shown in Section 3. The basic setup in ASDEX Upgrade discharge \#34575 is seen in Figure 1, and the procedures used for obtaining the experimental results follow what was outlined in Section 1: during the $B_{t}$-scan 200 pulses (each of $2 \mathrm{~ms}$ duration, which is very short compared with the $3.5 \mathrm{~s} \mathrm{du}-$ ration of the scan) with $\max \left(P_{0}\right) \approx 300 \mathrm{~kW}$ are injected in O-mode from the low-field side, using a gyrotron with $\omega_{0}=2 \pi \times 105 \mathrm{GHz}$ and fixed launcher settings. During each pulse, the electromagnetic scattering/emission signal in O-mode at frequencies close to $\omega_{0}$ is recorded using the fast acquisition CTS system, which also has a fixed receiver located on the low-field side, and power spectra are calculated by performing fast Fourier transforms on 655 ns windows of the recorded signal.

The mean power spectrum obtained during a pulse at $B_{t}=-2.531 \mathrm{~T}$, i.e. near the middle of the $B_{t}$-scan, is seen in Figure 2. In the figure, we clearly identify the gyrotron peak located inside a notch filter slightly below 105 $\mathrm{GHz}\left(\omega_{0}=2 \pi \times 104.93 \mathrm{GHz}\right)$, as well as the down-shifted high-frequency peaks excited by PDIs around $104 \mathrm{GHz}$. In addition to the down-shifted peaks, peaks up-shifted by a similar amount are seen; these peaks originate from scattering of the gyrotron wave by the low-frequency plasma waves excited by PDIs, and satisfy the selection rules

$$
\omega_{3}=\omega_{0}+\omega_{1}, \quad \mathbf{k}_{3}=\mathbf{k}_{0}+\mathbf{k}_{1} .
$$

Apart from the peaks, we note the frequency-dependent ECE background which indicates the expected decrease of radiation temperature with increasing frequency (decreasing major radius of the ECR location). For reference, we plot $\left(\omega_{0} \pm \omega_{1}\right) /(2 \pi)$ and $\left(\omega_{0} \pm \omega_{L H}\right) /(2 \pi)$ at the point where the PDI model of [8] leads to maximal amplification. Evidently, the simple estimate of the LH frequency underestimates the frequency shift of the PDI peaks relative to that of the gyrotron somewhat, while the warm plasma theory of [8] overestimates it somewhat; both these findings are robust throughout the $B_{t}$-scan, and can be attributed to finite plasma temperature and use of the dipole approximation, $\mathbf{k}_{0} \approx \mathbf{0}$, respectively [8]. The mean spectrum in Figure 2 is representative of those close to the onset of PDIs; for pulses later in the $B_{t}$-scan more peaks occur, but in this paper we shall only be concerned with whether or not PDI peaks occur at all. Making only this distinction, the $B_{t^{-}}$ scan separates into three regions in terms of the types of spectra observed: for $B_{t}<-2.557 \mathrm{~T}$ no spectra with PDI peaks are observed, for $B_{t} \in[-2.557 \mathrm{~T},-2.535 \mathrm{~T}]$ spectra with and without PDI peaks are observed intermittently, and for $B_{t}>-2.535 \mathrm{~T}$ all observed spectra contain PDI peaks; these observations are used for the comparison with the theory of [8] in Section 3.

\section{Modelling}

As mentioned in Section 2, the most important effect of the $B_{t}$-scan for PDI-purposes is to displace the ECR from inside the main plasma to the high-field side scrape-off layer, which should lead to a significant reduction of its optical thickness. This is indeed confirmed by calculations, based on [8], of the fraction of reflected power coupled to the plasma in X-mode, the fraction of power transmitted through the ECR from the gyrotron to the UHR (obtained by computing the optical thickness along the central beam rays, using a relativistic model described in $[16,17])$, and the total fraction of gyrotron power reaching the UHR (obtained by multiplying the two previous fractions), shown in Figure 3: the $\mathrm{X}$-mode fraction remains constant close to 0.1 until the end of the $B_{t}$-scan where it drops somewhat, while the fraction of power transmitted through the ECR increases from 0.1 at the beginning of the scan to 1 towards the end of the scan; the total fraction of gyrotron power reaching the UHR essentially follows the development of the transmitted fraction. We note a rather large uncertainty in the transmitted fraction due to uncertainties in the reconstructed magnetic equilibria from CLISTE [18], which determine the location of the last closed flux surface, as well as the electron temperature and density profiles from IDA [19], which vary rather quickly near the last closed flux surface and the values of which are primarily determined from measurements on the low-field side. We also note that Figure 3 only includes data points for pulses in which the central ray of the reflected X-mode beam reaches the UHR, which is necessary in order to apply the theory of [8].

To test the theory of [8] we compute $P_{0}^{\text {th }}$ (the gyrotron power necessary to excite PDIs) and plot the values obtained in Figure 4 . $P_{0}^{\text {th }}$ shows a development which is approximately the inverse of that of the total fraction from Figure 3 during the $B_{t}$-scan; this is as expected, since the conditions at the UHR are roughly similar throughout the $B_{t}$-scan (it remains well inside the main plasma) and the main variation is the amount of power transmitted from the 


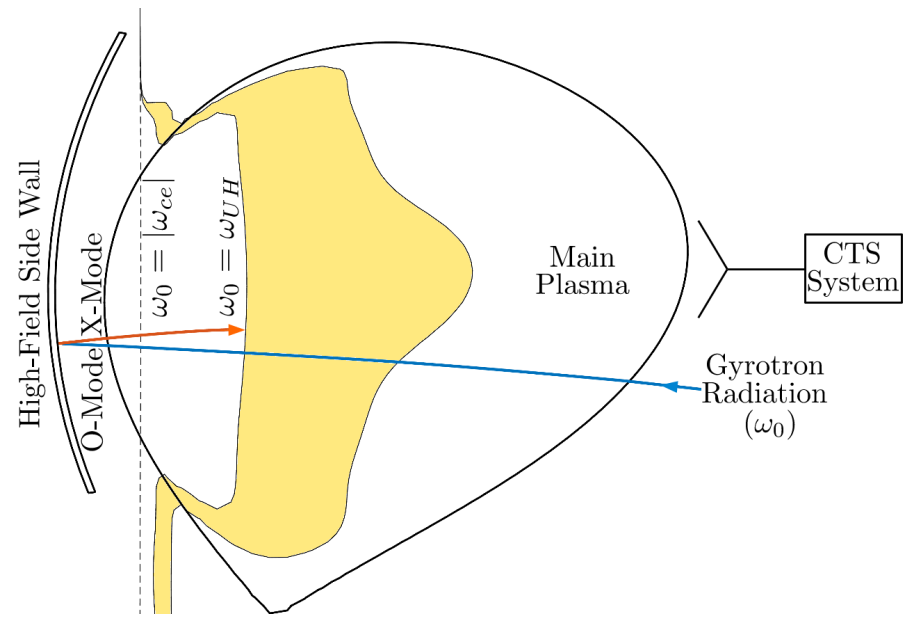

Figure 1. Experimental setup in ASDEX Upgrade discharge \#34575. Gyrotron radiation, with $\omega_{0}=2 \pi \times 105 \mathrm{GHz}$, is injected in O-mode from the low-field side, partially transmitted by the (optically thin) ECR $\left(\omega_{0}=\left|\omega_{c e}\right|\right)$ and reflected by the high-field side wall. After the reflection, part of the radiation re-enters the plasma in X-mode; if the ECR is not optically thick for the X-mode radiation, a significant amount of power may reach the UHR $\left(\omega_{0}=\omega_{U H}\right)$ where PDIs can be triggered due to field enhancement effects. The electromagnetic emission/scattering in O-mode at frequencies close to $\omega_{0}$ is measured using the fast acquisition CTS system. No propagating $\mathrm{X}$-mode radiation exists in the shaded region.

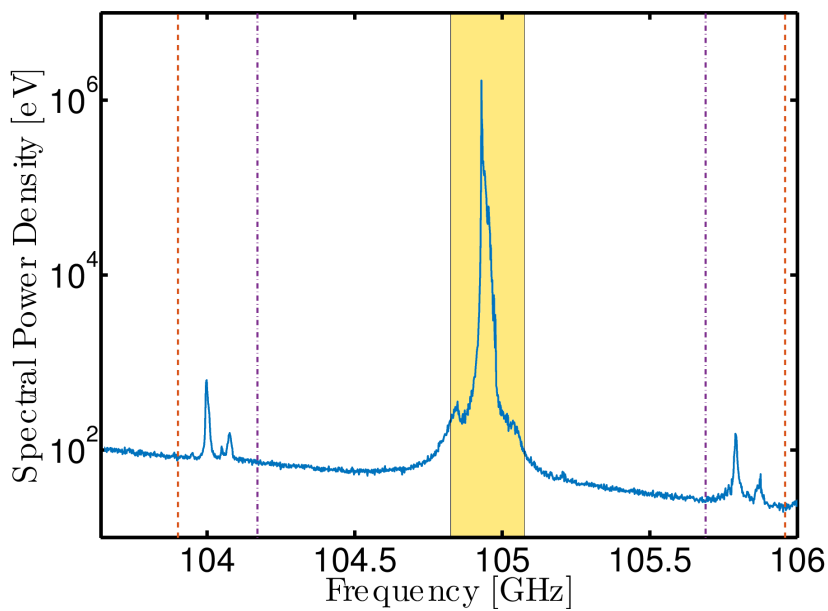

Figure 2. Mean spectrum recorded using the fast acquisition CTS system during a pulse at $B_{t}=-2.531 \mathrm{~T}$ in ASDEX Upgrade discharge \#34575. The gyrotron peak is clearly visible slightly below $105 \mathrm{GHz}\left(\omega_{0} /(2 \pi)=104.93 \mathrm{GHz}\right)$, and is located inside a notch filter stop band, marked by the shaded area, where the calibration giving the spectral power density in eV is rather uncertain. The peaks due to the PDI-generated plasma waves are shifted by $800-900 \mathrm{MHz}$, roughly $\omega_{L H} /(2 \pi)$, relative to the gyrotron peak; both down-shifted and up-shifted peaks are visible in the spectrum. For reference, $\left(\omega_{0} \pm \omega_{1}\right) /(2 \pi)$ and $\left(\omega_{0} \pm \omega_{L H}\right) /(2 \pi)$ from the theory of [8] are plotted as dashed and dashed-dotted lines, respectively; the peaks are located between the lines, as expected [8].

gyrotron to the UHR. In Figure 4 the pulses for which PDI peaks (and no PDI peaks) are observed are marked, along with $\max \left(P_{0}\right)=308 \mathrm{~kW}$. From [8], we expect that PDI peaks will be observed for $\max \left(P_{0}\right)>P_{0}^{\text {th }}$, and this condition is indeed seen to provide a good indicator for whether or not PDI peaks will be observed in Figure 4: for the 88 pulses with $\max \left(P_{0}\right)<P_{0}^{\text {th }}$, PDI peaks are only observed in 3 cases, while for the 61 cases with $\max \left(P_{0}\right)>P_{0}^{\text {th }}$, PDI peaks are only not observed in 5 cases. The theory of [8] thus seems to provide a quite reliable indication of whether or not PDI peaks will be observed for given gyrotron settings and plasma conditions, in spite of the large uncertainty on the fraction of power transmitted through the ECR.

\section{Conclusions}

We have investigated PDIs occurring for X-mode radiation near the UHR at ASDEX Upgrade. The PDIs were excited by injection of pulses of $105 \mathrm{GHz}$ gyrotron radiation in O-mode from the low-field side, which was transmitted by the optically thin ECR, reflected by the high-field side wall, and partially converted to X-mode. Part of the reflected X-mode power was transmitted through the ECR, whose optical thickness could be varied from a significant 


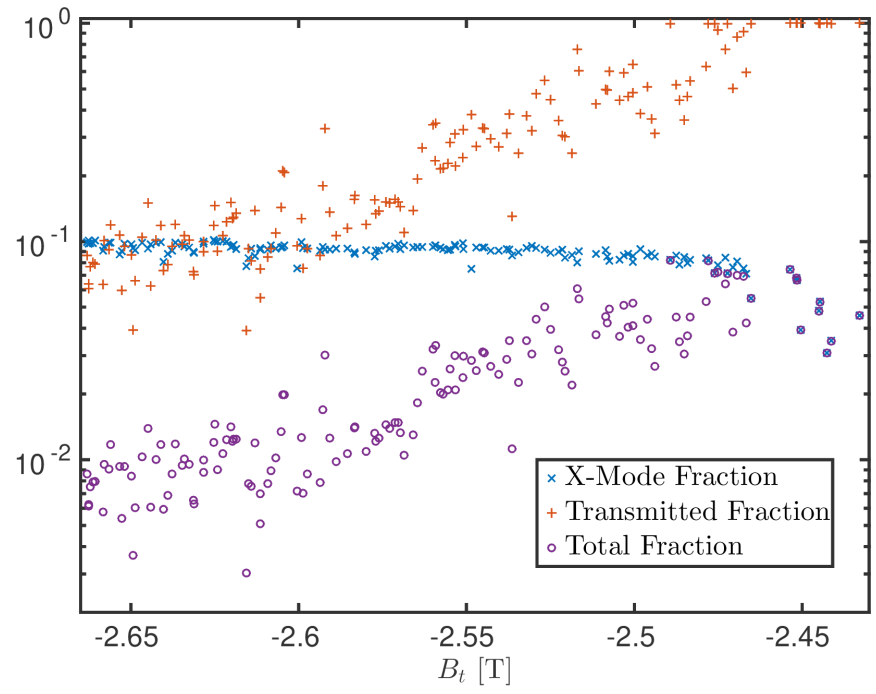

Figure 3. The fraction of reflected power coupled to the plasma in $X$-mode $(x)$, the fraction of power transmitted by the ECR from the gyrotron to the UHR (+), and the total fraction of gyrotron power reaching the UHR (o), during the $B_{t}$-scan in ASDEX Upgrade discharge \#34575, modelled using the theory of [8]. The X-mode fraction remains relatively constant with values around 0.1 throughout most of the scan. The transmitted fraction increases from approximately 0.1 to 1 during the $B_{t}$-scan; the relative change in the total fraction is similar to that of the transmitted fraction.

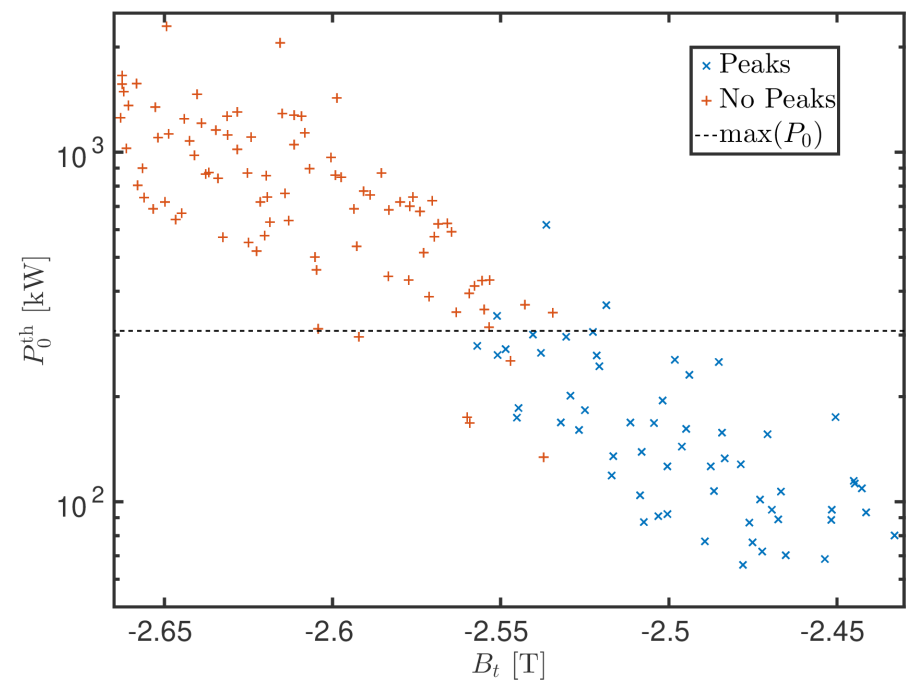

Figure 4. The development of $P_{0}^{\text {th }}$ during the $B_{t}$-scan in ASDEX Upgrade discharge \#34575, calculated using the theory of [8]. The pulses for which peaks are observed in the PDI frequency range (cf. Figure 2) are marked by $\times$, while the pulses for which no such peaks are observed are marked by $+; \max \left(P_{0}\right)=308 \mathrm{~kW}$ is marked by the dashed line. Evidently, the occurrence of PDI peaks is closely correlated with fulfilment of the condition $P_{0}^{\text {th }}<\max \left(P_{0}\right)$ provided by the theory of [8].

value to essentially zero by scanning $B_{t}$, and reached the UHR where it could excite PDIs due to field enhancement effects. The excitation of PDIs was observed by the occurrence of peaks shifted by roughly the LH frequency relative to the gyrotron peak in the spectra recorded using the fast acquisition CTS system. The occurrence of the PDI peaks was found to be well predicted by the theory of [8], which accounts for the fraction of power converted to X-mode at the wall reflection, the optical thickness of the ECR, field enhancement near the UHR, and models the PDI as decay of the X-mode waves into warm LH waves and electron Bernstein waves. Our findings in- dicate that the theory of [8] is capable of describing the onset of PDIs near the UHR in a magnetically confined fusion plasma, although it is only capable of providing limits of the frequency regions in which the PDI peaks occur due to the limitations of the dipole approximation on which it is based. It would thus seem reasonable to apply the theory of [8] to start-up scenarios for ITER in order to assess whether or not PDIs near UHR can be expected there; for the overdense plasmas expected in connection with $\mathrm{O}-\mathrm{X}-\mathrm{B}$ heating, the theory of [20] is presumably more appropriate. 


\section{Acknowledgements}

This work was supported by a research grant (15483) from VILLUM FONDEN. This work has been carried out within the framework of the EUROfusion Consortium and has received funding from the Euratom research and training programme 2014-2018 under grant agreement No 633053. The views and opinions expressed herein do not necessarily reflect those of the European Commission.

\section{References}

[1] M. Porkolab, Parametric Decay Instabilities in ECR Heated Plasmas, in Proc. 2nd Workshop Hot Electron Ring Physics (San Diego), edited by N.A. Uckan (National Technical Information Service, Alexandria, 1982), Vol. 1, p. 237

[2] F.S. McDermott, G. Bekefi, K.E. Hackett, J.S. Levine, M. Porkolab, Phys. Fluids 25, 1488 (1982)

[3] D.G. Bulyginsky, V.K. Gusev, V.V. Djachenko, M.A. Irzak, M.Yu. Kantor, M.M. Larionov, L.S. Levin, G.A. Serebreny, N.V. Shustova, ECR-Heating of Plasma in FT-1 Tokamak and Its Influence on the Ion Component, in Proc. 11th European Conf. Controlled Fusion and Plasma Physics (Aachen) (European Physical Society, Mulhouse, 1984), Vol. 1, p. 457

[4] Z.A. Pietrzyk, A. Pochelon, R. Behn, A. Bondeson, M. Dutch, T.P. Goodman, M.Q. Tran, D.R. Whaley, Nucl. Fusion 33, 197 (1993)

[5] R. Wilhelm, V. Erckmann, G. Janzen, W. Kasparek, G. Müller, E. Räuchle, P.G. Schüller, K. Schwörer, M. Thumm, the W VII-A team, Plasma Phys. Control. Fusion 26, 1433 (1984)

[6] S.K. Nielsen, P.K. Michelsen, S.K. Hansen, S.B. Korsholm, F. Leipold, J. Rasmussen, M. Salewski, M. Schubert, M. Stejner, J. Stober et al., Phys. Scr. 92, 024001 (2017)

[7] S.K. Hansen, S.K. Nielsen, M. Salewski, M. Stejner, J. Stober, the ASDEX Upgrade team, EPJ Web Conf. 149, 03020 (2017)
[8] S.K. Hansen, S.K. Nielsen, M. Salewski, M. Stejner, J. Stober, the ASDEX Upgrade team, Plasma Phys. Control. Fusion 59, 105006 (2017)

[9] S. Kubo, M. Nishiura, K. Tanaka, D. Moseev, S. Ogasawara, T. Shimozuma, Y. Yoshimura, H. Igami, H. Takahashi, T.I. Tsujimura et al., J. Instrum. 11, C06005 (2016)

[10] H.P. Laqua, V. Erckmann, H.J. Hartfuß, H. Laqua, W7-AS Team, ECRH Group, Phys. Rev. Lett. 78, 3467 (1997)

[11] H.P. Laqua, Plasma Phys. Control. Fusion 49, R1 (2007)

[12] V. Shevchenko, G. Cunningham, A. Gurchenko, E. Gusakov, B. Lloyd, M. O'Brien, A. Saveliev, A. Surkov, F. Volpe, M. Walsh, Fusion Sci. Technol. 52, 202 (2007)

[13] A. Köhn, G. Birkenmeier, A. Chusov, P. Diez, A. Feuer, U. Höfel, H. Höhnle, E. Holzhauer, W. Kasparek, S. Merli et al., Plasma Phys. Control. Fusion 55, 014010 (2013)

[14] D. Wagner, G. Grünwald, F. Leuterer, A. Manini, F. Monaco, M. Münich, H. Schütz, J. Stober, H. Zohm, T. Franke et al., Nucl. Fusion 48, 054006 (2008)

[15] M. Stejner, S. Nielsen, A.S. Jacobsen, S.B. Korsholm, F. Leipold, F. Meo, P.K. Michelsen, D. Moseev, J. Rasmussen, M. Salewski et al., Rev. Sci. Instrum. 85, 093504 (2014)

[16] H. Bindslev, Plasma Phys. Control. Fusion 33, 1775 (1991)

[17] H. Bindslev, Plasma Phys. Control. Fusion 34, 1601 (1992)

[18] P.J. McCarthy, P. Martin, W. Schneider, Tech. Rep. IPP 5/85, Max-Planck-Institut für Plasmaphysik, Garching b. München (1999)

[19] R. Fischer, C.J. Fuchs, B. Kurzan, W. Suttrop, E. Wolfrum, the ASDEX Upgrade team, Fusion Sci. Technol. 58, 675 (2010)

[20] E.Z. Gusakov, A.V. Surkov, Plasma Phys. Control. Fusion 49, 631 (2007) 